\title{
Study between elderly and adults of jequitinhonha valley: treatment adherence and prevalence of comorbidities
}

\begin{abstract}
The aims of this work are to verify the prevalence of the main comorbidities of the population in the city of Ponto dos Volantes, Minas Gerais; to assess the patients knowledge degree in relation to their diseases; to verify the association between knowledge of comorbidities, schooling, socioeconomic level and adherence to treatment; and to foster volunteering in the UFMG medical students. Eighty-seven patients, males and females, were selected from elderly and adults in the city of Ponto dos Volantes. The data collection was done by students of the Faculty of Medicine of the UFMG, allocated in outpatient clinics in the urban and rural area, during the visits, from July 24th to 29th, 2017. It was verified the adherence through the 8-item Morisky Medication Adherence Scale (MMAS-8). Statistical analysis was performed using the SPSS 18, Shapiro-Wilk, Student's T and Mann-Whitney tests. According to the national literature, arterial hypertension (75.3\%) and diabetes mellitus $(27.1 \%)$ are the most prevalent morbidities in the sample. Regarding the knowledge of the prescription and the meaning of the disease, $40.2 \%$ of patients did not understand the prescription and $49.4 \%$ of them did not understand the disease itself. Although there was no statistically significant difference, we identified low adherence in $24.4 \%$ of the elderly and in $14.3 \%$ of adults. The study was useful to evaluate the sociodemographic and comorbid profile of the population of Ponto dos Volantes, besides promoting health education and volunteering among students.
\end{abstract}

Keywords: ambulatory care, health centers, health literacy, morbidity surveys, treatment adherence and compliance, volunteers
Volume 4 Issue 6 - 2019

\begin{abstract}
Pedro Henrique de Almeida Andrade,' Maria Isabel Menezes Guedes,' Mariana América Gonçalves, 'Bruno Souto Rangel de Castro,' Gabriel Junqueira Lopes,' Gustavo Antunes Rodrigues Duarte,' Gustavo Couto Pereira da Silva,' Hugo Pimenta Ferreira,' Juliana Toledo Mesquita,' Maria Carolina Padovani Guerra,' Mateus Pinto Ribeiro,' Rafael Las Casas,' Rebeca Carolina Campos e Almeida Silva, ' Vitor Barbosa Abrantes,' Ana Paula Bernardes Real, ${ }^{2}$ Fernanda Mendes Amorim, ${ }^{2}$ Lívia Pires Calastri, ${ }^{2}$ Lucas Bastos Pereira Carneiro, ${ }^{2}$ Nathanna Fernandes Maciel, ${ }^{2}$ Fernando Henrique Pereira, ${ }^{3}$ Bruno Cézar Lage Cota, ${ }^{4}$ Luís Felipe José Ravic de Miranda ${ }^{4}$

'Medical student, Federal University of Minas Gerais, Brazil ${ }^{2}$ Physician, Federal University of Minas Gerais, Brazil ${ }^{3}$ Statistician, Federal University of Minas Gerais, Brazil ${ }^{4}$ Department of Internal Medicine, Federal University of Minas Gerais, Brazil
\end{abstract}

\begin{abstract}
Correspondence: Luís Felipe José Ravic de Miranda, Department of Clinical Medicine, Alfredo Balena Avenue number 190, Belo Horizonte, Minas Gerais, Brazil, Tel +5531984599393; Email ravicmirand@gmail.com
\end{abstract}

Received: November 28, 2019 | Published: December 20, 2019
Abbreviations: UFMG, universidade federal de minas gerais; MMAS-8, 8-item morisky medication adherence scale; DM, diabetes mellitus

\section{Introduction}

Arterial hypertension and Diabetes mellitus are the most prevalent chronic diseases in the world and in Brazil. These diseases have a high morbidity index, such as chronic kidney disease, visual changes, cardiovascular diseases and stroke., ${ }^{1,2}$

There is a prevalence of $21,4 \%$ of self-reported arterial hypertension $(\mathrm{AH})$ in between the Brazilian population. Higher rates are found at the southeast $(23,3 \%)$. Minas Gerais is the second highest in prevalence (24\%), after only the state of Rio Grande do Sul $(24,9 \%) .{ }^{3}$ Among the capitals, Belo Horizonte is the fourth in prevalence $(23,7 \%){ }^{4}$

Regarding diabetes mellitus (DM), there is a national prevalence of $6,2 \%$ and again, the southeast has the higher prevalence (7\%). The State of Minas Gerais and the city of Belo Horizonte has a prevalence of $6,5 \%{ }^{5}$

A 2012 study, in which 447 patients, diagnosed with $\mathrm{AH}$, were asked about the information of their disease, found these patients had insufficient knowledge and they had uncontrolled hypertension, indicating that physicians should place greater emphasis on patients about target blood pressure and the importance of correct use of antihypertensive drugs. ${ }^{6}$

Also, a study published by Jankowska-Polańska et al. ${ }^{7}$ in which 602 patients with hypertension were analyzed for disease acceptance and treatment adherence using the Acceptance of Illness Scale (AIS) and the Morisky Medication Adherence Scale (MMAS), showed that disease acceptance correlates with adherence to pharmacological treatment. Therefore, general assessment of disease acceptance should be considered in daily clinical practice. ${ }^{7}$ 
Verbal and written information is important for better patient understanding about their treatment. Moreover, clear, simple and attractive reading leaflets significantly improve patient knowledge, being particularly important in emergencies. Good doctor-patient relationship is critical to allow patients to better understand their illness, increase the patient confidence in doctors and improving treatment adherence. ${ }^{8,9}$

The present study is an evaluation of the prevalence of noncommunicable chronic comorbidities such as AH and DM, among others, in a group of people from Ponto dos Volantes (PV), with approximately 15.000 inhabitants, comparing national, state and municipal data on health situation. The city is located in the Jequitinhonha Valley, a region which has the lowest HDI (human development index) of Minas Gerais. The data on the health care of inhabitants of PV are insufficient. This study will allow a better knowledge about health region, favoring a public policy with greater relevance and property.

\section{Material and methods}

A cross-sectional study was performed in PV. Data was collected by nineteen Medicine students from Faculty of Medicine of UFMG. They were allocated in several 'out-patients' clinics from the urban and rural areas to evaluate patients from 24th to 29th of july of 2017. The students were supervised and oriented by two professors from the Department of Internal Medicine of Medicine School of UFMG.

The project was approved by UFMG's ethics and research committee and inserted in "Plataforma Brasil" under the number 76797317.8.0000.5149. Informed consent was obtained from all participants.

The recruitment of patients was made by city hall's and health professional's disclosure. All patients included were shared in two groups: adults (18 to 59 years) and older people (60 years or more). People younger than eighteen years-old and those who did not give their consent to use their data were excluded from the sample.

The project was composed of three phases: pre-consultation, consultation and educational lectures. The pre-consultation and consultation were done when patients looked for appointments with the doctors. The educational lectures, in number of four, (arterial hypertension, diabetes mellitus, smoking/DPOC and depression) were given in four different days during the period from 24th to 27th of July, 2017.

During the pre-consultation, patients were evaluated about smoking habits (Fagërstrom test), drinking (daily dose, type of drink, time of consumption, CAGE questionnaire) and feeding (food intake diary). Social level was assessed according to the Economic Classification Criteria of Brazil - 2015 and, when it was necessary, Mini-Mental State Examination was applied to assess cognitive impairment.

After that, the patients went to the consultation and the students made the anamnesis, physical exam, cognitive test (if indicated) and requested laboratorial exams (if necessary)

In order to verify the adherence, it was used the 8-item Morisky Medication Adherence Scale (MMAS-8) written by Morisky DE, Green LW, Levine DM (1986). The MMAS-8 is the method used to evaluate the medication adherence according to the patient's answer (never, almost never, sometimes, often, always) for each of the eight questions. The score is made according to each answer and verified the adherence degree with the total score. It was considered low medication adherence values below six points; medium medication adherence between six and seven points and high medication adherence values above eight points. After that, it was done a questionnaire for patients who did not reach eight points or more, in order to investigate the reasons for the medium and low medication adherence. Some of the reasons were: not understanding the disease and/or medications, having a neurological or cognitive deficit, they think it is not necessary to take the medications, to forget to take the medications, to doubt the medication will improve the clinical status, to take a lot of medications and others.

The third phase was composed of educational lectures which took place in the primary health care services. They were given by students and supervised by professors. Data was collected and an Excel spreadsheet was created to record patient data.

All data were analyzed using SPSS version 18 software and at a significance level of 0.05 . To verify the hypothesis of association between two categorical variables, Pearson's chi-square test was applied or Fisher's exact test when the assumptions of the first test were not satisfied.

\section{Results}

Eighty-seven patients were analyzed, in which thirty $(34,5 \%)$ were adults and fifty-seven $(65,5 \%)$ elderly. The average age was 64,46 years old (24-99) and a median of 66 years old. Regarding gender, $87,(60 \%)$ were female. About the marital status, $35(40,2 \%)$ were married, 27 (31\%) widowed, 17 (19,5\%) single, 5 (5,7\%) divorced and $3(3,4 \%)$ had no data collected. About the color of skin, $25(28,7 \%)$ patients considered themselves as white $34(39,1 \%)$ considered themselves as having an intermediary color of skin between white and black people, $20(23 \%)$ considered themselves as black and $8(9,2 \%)$ did not related their color.

Literacy status resulted as follows: 4,8\% were illiterate, while $26,4 \%$ had incomplete elementary school; $14,9 \%$ had complete elementary school; $2,3 \%$ had incomplete high school; $6,9 \%$ had finished their high school; only $2,3 \%$ had complete higher education and 2,3\% did not have their schooling documented, as illustrated in Figure 1. Also, concerning the economic classification, 5,7\% were classified as C1 class, $20,7 \%$ as $\mathrm{C} 2$ class and $73,6 \%$ as D-E classes.

Among the comorbidities evaluated (Figure 2): 64 (75,3\%) of the patients had AH, $23(27,1 \%)$ had DM, $18(21,7 \%)$ had cataract, $14(16,7 \%)$ dyslipidemia, $12(14,5 \%)$ gastroesophageal reflux disease, $12(14,1 \%)$ cholelithiasis, $11(13,1 \%)$ drinking, $10(11,9 \%)$ major depressive disorder, $6(7,2 \%)$ peripheral vascular disease, $6(7,1 \%)$ cardiac insufficiency, $6(7,1 \%)$ previous stroke event, $5(5,9 \%)$ smoking, $4(4,7 \%)$ neoplasia, $2(2,4 \%)$ some form of coronary artery disease and only one $(1,2 \%)$ chronic obstructive pulmonary disease (COPD). It is worth mentioning that the prevalence of comorbidities was evaluated according to patients' report. The association between number of comorbidities and age range is demonstrated in Table 1.

It was asked if the patients understood their own disease and the medical prescription in order to evaluate, indirectly, how effective were medical orientations and explanations. The results were: 35 of them did not understand the prescription and $43(49,4 \%)$ did not understand their own disease versus $46(52,9 \%)$ that did understand the prescription and $2(40,3 \%)$ that did understand their disease. Six patients $(6,9 \%)$ did not have their comprehension about the prescription 
documented and nine $(10,3 \%)$ did not have their comprehension about their disease documented, as illustrated in Figure 3.

Regarding the medication adherence, measured by the MMAS8 scale, the patients were divided between low, medium and high medication adherence. Among the patients with low adherence, the majority were elderlies (24,4\% from the total of elderlies and $14,3 \%$ from the total of adults). Among the ones with medium and high adherence the difference between elderlies and adults was smaller as seen in (Tables 1) (Table 2).

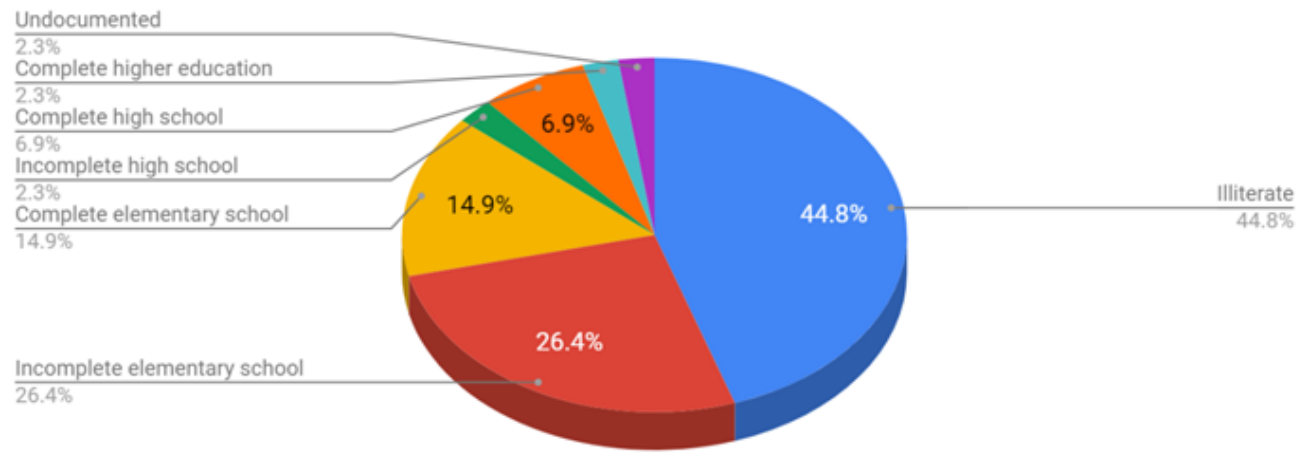

Figure I Educational level in patients from Ponto dos Volantes.

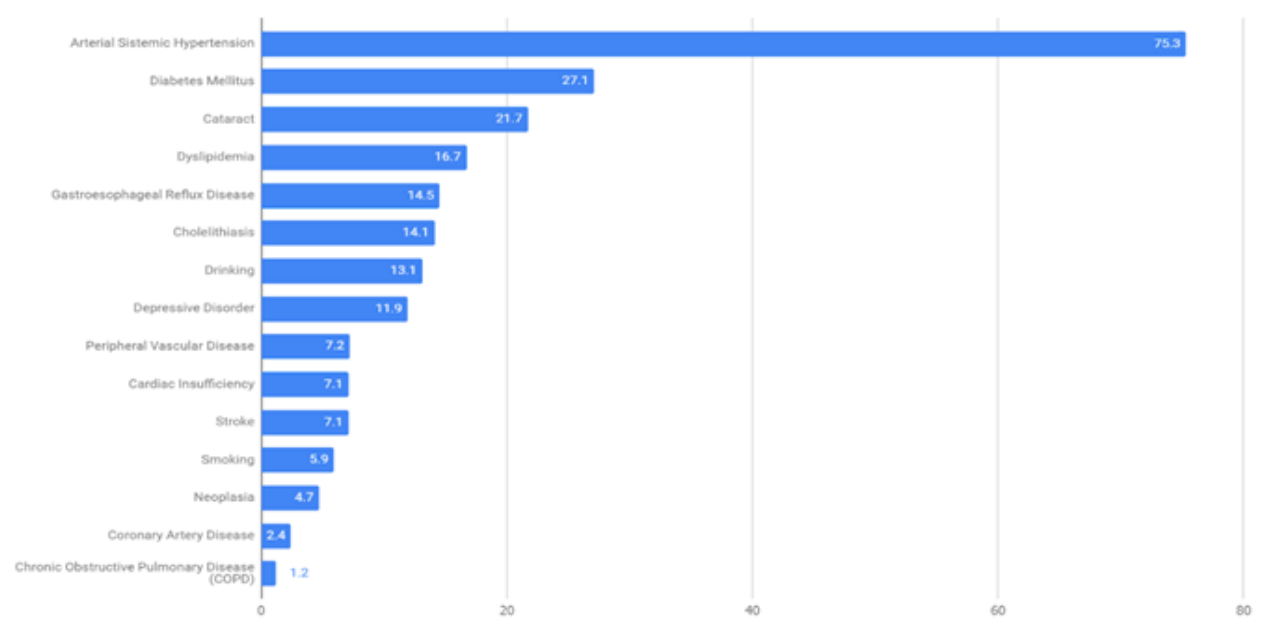

Figure 2 Prevalence of Comorbidities in patients from Ponto dos Volantes.

Not $\square$ Yes

60

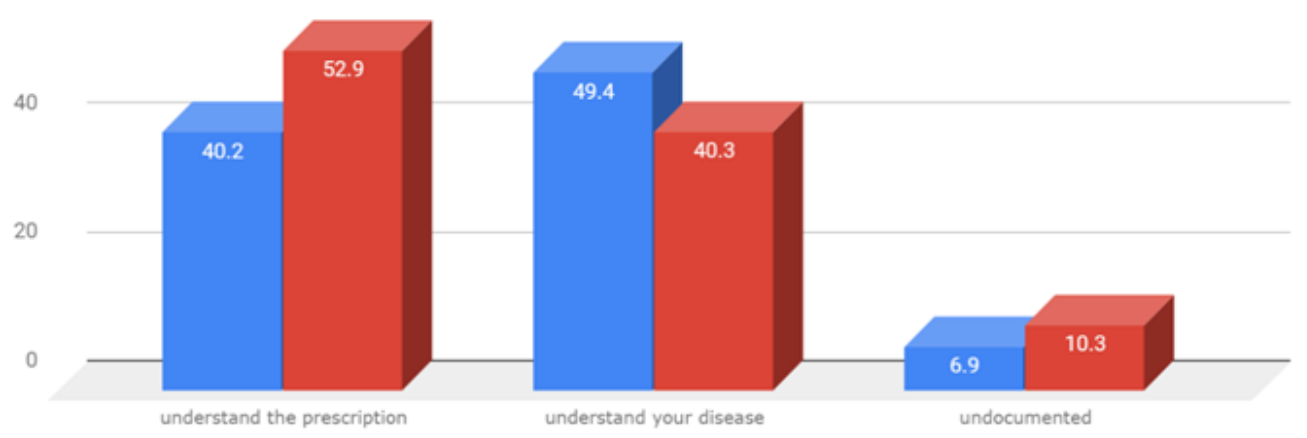

Figure 3 Percentage of patients who understood their disease and prescription in the city of Ponto dos Volantes. 
Table I Association between number of comorbidities and age range (adults versus elderlies)

\begin{tabular}{lllll}
\hline Number of comorbidities & Adults & Elderlies & Total & P value \\
\hline 0 & $4(13,3 \%)$ & $3(5,3 \%)$ & $7(8,0 \%)$ & 0,730 \\
1 & $14(46,7 \%)$ & $26(45,6 \%)$ & $40(46,0 \%)$ & \\
2 & $6(20,0 \%)$ & $18(31,6 \%)$ & $24(27,6 \%)$ & \\
3 & $5(16,7 \%)$ & $9(15,8 \%)$ & $14(16,1 \%)$ & \\
4 & $0(0 \%)$ & $1(1,8 \%)$ & $1(1,1 \%)$ & \\
5 & $1(3,3 \%)$ & $0(0,0 \%)$ & $1(1,1 \%)$ & \\
Total & $30(100 \%)$ & $57(100 \%)$ & $87(100 \%)$ & \\
\hline
\end{tabular}

Table 2 Medication adherence according to age range

\begin{tabular}{llllll}
\hline Groups & Low adherence & Medium adherence & High adherence & Total & p value \\
\hline Adults & $3(14,3 \%)$ & $7(33,3 \%)$ & $1 \mathrm{I}(52,4 \%)$ & 21 & 0,586 \\
Elderlies & $11(24,4 \%)$ & $13(28,9 \%)$ & $21(46,7 \%)$ & 45 \\
Groups & $14(21,2 \%)$ & $20(30,3 \%)$ & $32(48,5 \%)$ & 66
\end{tabular}

\section{Discussion}

Drawing the health profile of a population is an important element to use in the coordination and improvement of care actions. Analysis of the present study showed that $69 \%$ of patients are women. The predominant age group was elderly $(65,5 \%)$, with a mean age of 64,46 years. The high proportion of illiterate people in the sample $(44,8 \%)$ was consistent with the latest data from municipality, which showed illiteracy rate among 60 to 69 years old $(68,8 \%) .{ }^{10}$ The Jequitinhonha Valley illiteracy rates are the highest in the state. Among the most prevalent comorbidities in the sample are AH first $(75,3 \%)$ and DM second $(27,1 \%)$, a profile consistent with the Brazilian population, but at higher levels considering a prevalence of $\mathrm{AH} 21,4 \%$ and DM $6,2 \%$ in Brazil. ${ }^{3,4}$ A possible determinant for a higher prevalence of $\mathrm{AH}$ in this population is the fact that many patients are monitored at the primary health care due to hypertension. Regarding therapeutic adherence, the patients with low adherence were mostly elderly $(24,4 \%$ of the total elderly and $14,3 \%$ of the total adults). Among those with medium and good adherence, the elderly-adult difference was smaller. $46,7 \%$ of the elderly and $52,4 \%$ of the adults were classified as having good adherence.

In a study with a hypertensive population in Rio Grande do Sul, Gewehr et al. ${ }^{11}$ reported that in the age group 55-64 years old, $73,8 \%$ of individuals had good adherence. On the other hand, among individuals over 64 years old, $60 \%$ were classified as having low adherence. Other factors that decreased adherence in this study were lower family income (4,7 times more likely to have low adherence) and being single. In a study to evaluate adherence to the influenza vaccination campaign in Campinas-SP, the coverage of vaccination was $74 \%$ among the elderly, not assessing age interference in adherence..$^{12}$ Another study, which evaluated medication adherence among the elderly in outpatient follow-up also in Campinas-SP, indicated adherence of $88.5 \%$ among these elderly. ${ }^{13}$ These data corroborate other investigations in the elderly population that identified high adherence rates in this population. ${ }^{9}$ Considering the literature, we note a result of $46.7 \%$ of good adherence among the elderly in PV, which means, a low value, suggesting that other variables contributed to this result.

According to Kauser et al. ${ }^{14}$ marital status, socioeconomic status, and educational level, such as illiteracy, were associated with a higher level of non-adherence compared to those with a marital relationship and undergraduate education. Due to these data, we realized that a population with a predominance of illiteracy (45\%) and of the classes D-E $(73 \%)$ as the present study presented high vulnerability and probable poor adherence. The proportion of illiterate people from PV aged 40 to 59 years old was $36.7 \%$ in $2010,{ }^{10}$ considerably lower than the number of illiterate elderlies on the same date and location. This is one of the factors that could explain the difference in adherence between the two populations. There is a big gap between prescribing the medication, making sure that the patient understood the explanation given by the doctor and taking the medication correctly. In this study, $40.2 \%$ of patients said they did not understand their prescription and $49.4 \%$ said they did not understand their own disease. Jackson et al. ${ }^{15}$ correlated poor medication adherence with low literacy, forgetfulness, high cost of medication, limited access to care, poor patient-physician communication, lack of confidence in the physician and depression. Moreover, in this study, most patients with low adherence were elderly (Table 2). Some authors call the knowledge of diseases as health literacy. This term is mainly understood as an individual characteristic that concerns the ability to obtain, process and understand basic health information in order to effectively navigate the health system. ${ }^{16}$ Patients with low health perceive low self-efficacy in relation to your health conditions; are not willing to participate in care delivery; present higher risks of hospitalization and mortality; and are not aware of the determinants of well-being. Thus, the limitation of health literacy has been associated with inadequate management of long-term conditions. 
Vermeire et al ${ }^{17}$ concluded that the patient's relationship with the health team and especially with the doctor is one of the factors that most impact treatment adherence. Good communication that informs about treatment benefits, dosage instructions and adverse effects encourages and strengthens adherence. When communication fails, either by the health team or by cognitive and memory problems, which mainly affect the elderly population, adherence is compromised. Among the factors related to patients, one of the factors that most impact adherence is cognitive limitations, physical disability or decreased mobility. Among the elderly of PV, where there were high rates of illiteracy, there was a reduction in cognitive and communication skills of patients, beyond that expected for age. Among adults with higher levels of education, we believed that communication is more effective and enlightening, which helps explain the difference in adherence.

In addition, more than two comorbidities in PV were more common in the elderly than in adults. Osterberg et al. ${ }^{18}$ pointed to a therapeutic regimen with several medications or several daily doses as being negative for adherence. Therefore, in the older population of $\mathrm{PV}$, which has the highest number of comorbidities, the drug regimen is expected to be more complex and having lower adherence.

Osterberg et al. ${ }^{18}$ concluded that health literacy is not associated with self-reported or objectively measured medication adherence in patients treated with type $2 \mathrm{DM}$, but that lower health literacy scores are associated with patients with having difficulty remembering to take medications. As memory resources are applied to remind them this task, i.e. to take their medication, there is therefore an improved adherence to treatment. Van Onna et al. ${ }^{19}$, however, showed that poor health literacy is correlated with patient's non-adherence to treatment and that they often recognized these knowledge gaps. It also showed that one of the reasons for noncompliance is that health professionals provide conflicting messages about medication and that half of patients, especially at the time of diagnosis, would like to know more about the cause of the disease, treatment goals and the long-term consequences

The present study had some limitations, such as the cross-sectional design, with a brief data collection period (one week), as well as a relatively small sample size $(\mathrm{n}=87)$ compared to other similar studies. Moreover, the sample was obtained by patients who were seen in a primary health care setting, not from the general population.

Further studies are needed to better assess the population profile of the region, preferably with a larger number of subjects, and, if possible, with longitudinal follow-up of patients. These factors reduce the likely impact of some of the biases described here, allowing greater precision for the correlation estimates among variables, such as: education, knowledge of comorbidities, socioeconomic level and adherence to treatment.

\section{Conclusion}

The importance of the study rests upon the first turn analysis of the social-demographic profile and prevalent comorbidities of the population from Ponto dos Volantes, in the Jequitinhonha Valley, which were not documented yet. Moreover, it discovers the reasons for low therapeutic adherence.

The present project was able to promote volunteering among the students and to provide the knowledge about diseases, correct treatment and means to prevent complications to the patients and healthy inhabitants. Also, it was able to analyze the consequences of our actions in order to make possible future interventions in public health.

\section{Funding details}

None.

\section{Acknowledgments}

None.

\section{Conflicts of interest}

The authors declare that there is no conflicts of interest.

\section{References}

1. Ministry of health, health surveillance secretariat, health situation analysis department, strategic action plan for the fight against chronic noncommunicable diseases (ncds) in Brazil (2011-2022). Ministry of Health; 2011.

2. Alwan A, Maclean DR, Riley LM, et al. Monitoring and surveillance of chronic non-communicable diseases: progress and capacity in highburden countries. Lancet. 2010;376(9755):1861-1868.

3. Andrade S, Stopa S, Brito A, et al. Prevalence of self-reported arterial hypertension in the Brazilian population: analysis of the national health survey, 2013. Epidemiol Serv Saúde. 2015;24(2):297-304.

4. Schmidt M, Duncan B, Hoffmann J, et al. prevalence of diabetes and hypertension in Brazil based on self-reported morbidity survey, Brasil, 2006. Rev Saúde Pública. 2009;43(2):S74-S82.

5. Iser B, Stopa S, Chueiri P, et al. Prevalence of self-reported diabetes in Brazil: results from the National Health Survey 2013. Epidemiol Serv Saúde. 2015;24(2):305-314.

6. Almas A, Godil S, Lalani S, et al. Good knowledge about hypertension is linked to better control of hypertension: A multicentre cross sectional study in Karachi, Pakistan. BMC Res. Notes. 2012;530-579.

7. Jankowska-Polańska B, Chudiak A, Uchmanowicz I, et al. Selected factors affecting adherence in the pharmacological treatment of arterial hypertension. Patient Preference and Adherence. 2017;11:363-371.

8. Mavri A, Ostaseski FN, Kramaric A, et al. New educational approach for patients on warfarin improves knowledge and therapy control. Wien Klin Wochenschr. 2015;127(11-12):472-476.

9. Tzaneva V, Iacob T. A better comunication with the patients improves the management of HIV disease: a nonsystematic review. Clujul Med. 2013;86(3):181-184.

10. Taxa de analfabetismo segundo município [Internet]. 2019

11. Gewehr D, Flag V, Gelatti G, et al. Adherence to pharmacological treatment of hypertension in Primary Health Care. Saude em Debate. 2018;42(116):179-190.

12. Francisco P, Borim F, Neri A. Influenza vaccination in the elderly: FIBRA data, Campinas, São Paulo, Brasil. Ciência \& Saúde Coletiva. 2015;20(12):3775-3786

13. Cintra F, Guarient M, Miyasaki L. Adesão medicamentosa em idosos em seguimento ambulatorial. Ciência \& Saúde Coletiva. 2010;15 Suppl 3:S3507-S3515

14. Kumaraswamy RC, Kauser MM, Jagadeesh MK, et al A. Study of determinants of nonadherence to anti-hypertensive medications in essential hypertension at a teaching hospital in Southern India. CHRISMED J Health Res. 2015;2(1):57-60. 
15. Jackson IL, Adibe MO, Okonta MJ, et al. Medication adherence in type 2 diabetes patients in Nigeria. Diabetes Technol Ther. 2015;17(6):398 404.

16. Palumbo R. Discussing the effects of poor health literacy on patients facing HIV: a narrative literature review. Int J Health Policy Manag. 2015;4(7):417-30.

17. Vermeire E, Hearnshaw H, Van Royen P, et al. Patient adherence to treatment: three decades of research. A comprehensive review. J Clin Phar. Ther. 2001;26(5):331-342.
18. Osterberg L, Blaschke T. Adherence to medication. $N$ Engl $J$ Med. 2005;353(5):487-497.

19. Van Onna M, Hinsenveld E, de Vries H, et al. Health literacy in patients dealing with gout: a qualitative study. Clin Rheumatol. 2014;34(9):1599 1603 . 